\title{
Criminologie
}

\section{Redresser les torts : l'abolitionnisme et le contrôle de la criminalité}

\section{Willem de Haan}

Volume 25, numéro 2, 1992

Nouvelles connaissances et nouvelles questions en criminologie

URI : https://id.erudit.org/iderudit/017325ar

DOI : https://doi.org/10.7202/017325ar

Aller au sommaire du numéro

\section{Éditeur(s)}

Les Presses de l'Université de Montréal

ISSN

0316-0041 (imprimé)

1492-1367 (numérique)

Découvrir la revue

Citer cet article

de Haan, W. (1992). Redresser les torts : l'abolitionnisme et le contrôle de la criminalité. Criminologie, 25(2), 115-137. https://doi.org/10.7202/017325ar
Résumé de l'article

In this article 'abolitionism' will be discussed as a social movement, a theoretical perspective, and a political strategy. Strategies for penal reform will be dealt with and the implications of the abolitionist perspective for crime control will be discussed. As a theoretical perspective, abolitionism takes on the twofold task of providing a radical critique of the criminal justice system while showing that there are other, more rational ways of dealing with crime. It will be argued that what is needed is a wide variety o social responses rather than a uniform state reaction to the problem of crime. Therefore, a reconceptualization of the notions of crime and punishment is offered in the form of the concept of redress. In policy terms it is claimed that social policy instead of crime policy is needed in dealing with the social problems and conflicts that are currently singled as the problem of crime. 
In this article 'abolitionism' will be discussed as a social movement, a theoretical perspective, and a political strategy. Strategies for penal reform will be dealt with and the implications of the abolitionist perspective for crime control will be discussed. As a theoretical perspective, abolitionism takes on the twofold task of providing a radical critique of the criminal justice system while showing that there are other, more rational ways of dealing with crime. It will be argued that what is needed is a wide variety o social responses rather than a uniform state reaction to the problem of crime. Therefore, a reconceptualization of the notions of crime and punishment is offered in the form of the concept of redress. In policy terms it is claimed that social policy instead of crime policy is needed in dealing with the social problems and conflicts that are currently singled as the problem of crime.

L'expression «abolitionnisme" désigne à la fois un mouvement social, une stratégie politique de réforme et une perspective théorique. L'abolitionnisme, en tant que mouvement social, est concerné par l'abolition des prisons, voire du système pénal ${ }^{2}$ dans son ensemble, et prend ses origines dans les luttes pratiques pour les droits des personnes incarcérées et pour la réforme pénale. L'abolitionnisme, en tant que stratégie politique, se fonde sur une analyse des réformes pénales et propose différentes manières de prendre des positions à leur égard. En tant que perspective théorique, l'abolitionnisme se donne la double tâche d'articuler une critique radicale du système pénal tout en montrant en même temps qu'il existe d'autres manières plus rationnelles de faire face au problème de la «criminalité». Bref, l'abolitionnisme part de la conviction que la vie sociale ne doit pas, et ne peut pas dans les faits, être réglée effectivement par le droit criminel. Dès lors, le rôle du système de justice criminelle doit être réduit de façon draconienne, tandis que d'autres manières de résoudre les situations problématiques doivent être mise en place progressivement.

1. Professeur, Rijkuniversiteit te Utrecht, Willem Pompe Instituut voor Strafrechtswetenschappen, Janskerkhof 16, 3512 BM Utrecht, The Netherlands.

2. L'expression «système pénal " est utilisée ici dans son acception large de manière à inclure aussi le droit pénal. 
Dans cet article, nous nous pencherons sur la perspective abolitionniste dans le cadre de ce triple point de vue. Nous examinerons tout d'abord l'abolitionnisme en tant que mouvement de réforme pénale. Puis, nous nous intéresserons aux différentes stratégies pour réaliser une réforme pénale ainsi qu'aux répercussions de la perspective abolitionniste sur le contrôle de la criminalité. Ensuite, nous regarderons l'abolitionnisme en tant que perspective théorique et critique de la criminalité et de la peine. Nous avancerons l'idée que, ce qu'il faut, c'est une grande variété de réponses sociales plutôt qu'une réaction étatique uniforme au problème de la criminalité. Quant à la politique à adopter, nous verrons qu'une politique sociale répondrait mieux qu'une politique criminelle aux problèmes et aux conflits sociaux qui sont actuellement regroupés sous une même enseigne, à savoir la notion de criminalité. Enfin, nous proposerons un nouveau concept pour approcher la question de la criminalité et de la peine: celui de redressement.

\section{L'ABOLITIONNISME EN TANT QUE MOUVEMENT SOCIAL}

À la fin des années soixante, un besoin impérieux de déstructurer le contrôle social de la déviance et de la criminalité s'est emparé des courants de pensées (Cohen, 1985). C'est alors que l'abolitionnisme est apparu comme un mouvement pour l'abolition de la peine de prison. Ainsi, en Europe occidentale, des groupes opposés à l'incarcération se formaient en Suède et au Danemark (1967), en Finlande et en Norvège (1968), en Grande-Bretagne (1970), en France (1970) et aux Pays-Bas (1971). Leur principal objectif était d'adoucir les souffrances que la société inflige aux prisonniers. Cela passait par une profonde mutation de la conception du châtiment, une humanisation des différentes formes d'incarcération au court terme, et à plus long terme, le remplacement du système pénitentiaire par des mesures plus appropriées et plus actualisées de contrôle de la criminalité.

Il a été avancé que les mouvements abolitionnistes voient généralement le jour dans les petits pays ou dans des pays qui connaissent un faible taux de criminalité et «would never have been «invented» in a country like the United States of America with its enormous crime rate, violence, and criminal justice apparatus» (Scheerer, 1986, p. 18). Toutefois, au Canada et aux États-Unis, les familles d'(anciens) prisonniers, des groupes religieux et des individus se consacraient également à des activités de soutien aux détenus et militaient activement pour une réforme du système carcéral. Ainsi, les partisans de l'abolition de la 
peine de prison aux États-Unis considèrent leur lutte comme une mission historique qui perpétue et couronne la lutte de leurs ancêtres contre l'esclavage. Pour eux, l'emprisonnement est une forme de blasphème, soit un acte moralement répréhensible et indéfendable qui doit donc être aboli (Morris, 1976, p. 11). C'est dans cette perspective qu'on propose alors une stratégie à long terme: une guerre d'usure en trois actes. Tout d'abord, le gel complet de la planification et de la construction de prisons; ensuite, l'exclusion de certaines catégories de contrevenants du système carcéral et, enfin, la décarcération, soit la libération d'un maximum de détenus. Puisant ses sources dans les mouvements de réforme des prisons des années soixante et soixante-dix aussi bien en Europe occidentale qu'en Amérique du Nord, l'abolitionnisme s'est imposé comme un nouveau paradigme en criminologie (critique) et comme une nouvelle approche du contrôle de la criminalité (Bianchi et Van Swaaningen, 1986; Lasocik, Platek et Rzeplinska, 1991).

Pour les abolitionnistes, les États-Unis constituent l'exemple parfait d'un pays qui souffre des conséquences d'une obsession pour le châtiment. L'adoption d'une politique de contrôle de la criminalité plus sévère s'est traduite par un accroissement du nombre de personnes incarcérées pour des périodes de temps plus longues. On assiste donc à une augmentation dramatique du nombre de détenus qui sont passés d'environ 350000 dans les années soixante-dix à 850000 à Ia fin des années quatre-vingt. Près de $80 \%$ des augmentations dans les admissions à la prison sont liées à la drogue. Au mois de septembre 1988, environ $44 \%$ de tous les prisonniers fédéraux avaient été incarcérés pour des violations des lois sur les stupéfiants. Selon les prévisions du National Council of Crime and Delinquency Prison Population de 1989, la "guerre contre la drogue" aura pour effet de gonfler davantage encore la population des détenus entre 1989 et 1994 . On retient le chiffre de plus de $68 \%$, soit un total de 1133000 détenus dont les personnes de couleur constituent toujours la majorité écrasante. Avec un taux de 440 prisonniers par 100000 habitants, les États-Unis consacrent leur suprématie en matière de recours à l'incarcération. Même avec un taux d'incarcération passant de 30 en 1980 à environ 50 vers le milieu des années quatre-vingt-dix, les Pays-Bas demeureront au plus bas de l'échelle. Et pourtant, cette politique moins répressive ne rend pas le problème de la criminalité plus grave aux Pays-Bas qu'aux États-Unis.

À l'instar des États-Unis, aux Pays-Bas on considère la «criminalité de rues» comme un problème social majeur. De fait, la première enquête sur la criminalité dans le monde (International Crime Survey) 
(Van Dijk, 1990) révélait que les taux de victimisation entre 1983 et 1988 étaient plus élevés aux États-Unis et aux Pays-Bas que dans tous les autres pays considérés. On notait toutefois des différences fondamentales dans la gravité des infractions et dans l'efficacité des mesures de contrôle. Les taux de victimisation étaient certes aussi élevés dans les deux cas, mais, aux Pays-Bas, ils s'expliquaient par l'exceptionnelle prévalence des vols de bicyclettes alors qu'aux États-Unis il s'agissait surtout d'homicides, de vols, et d'agressions (sexuelles).

S'il faut en tirer une conclusion, c'est que la relation entre la criminalité et son contrôle par l'incarcération est bien plus complexe que les partisans de l'incarcération semblent penser. L'approche punitive aux problèmes reliés à la criminalité a peu à offrir en termes de protection de la société et, s'il est impossible de jamais gagner la "guerre contre la drogue», elle s'accompagne hélas de graves conséquences.

En effet, dans son ensemble, le système carcéral, qui produit des résultats contraires à ceux qu'on attend, est difficile à contrôler et constitue lui-même un des principaux problèmes sociaux. C'est pourquoi les abolitionnistes rejettent l'idée que le système de justice pénale ait un rôle à jouer en matière de protection. Ils sont d'ailleurs pessimistes quant à la capacité du droit pénal de résoudre les conflits. Selon eux, loin de l'améliorer, le système pénal actuel aggrave plutôt la situation.

Dans la «guerre contre la drogue » que mènent les États-Unis ainsi que de nombreux autres pays, on doit souvent faire appel à des techniques d'appréhension des suspects qui posent un problème éthique et sur lesquelles on ferme les yeux - quand on ne les considere pas comme nécessaires. Or, cela favorise l'apparition de différentes formes de complicités organisationnelles qui minent encore plus la légitimité du système de justice pénale, déjà discutable. Selon Roshier (1989), la "guerre contre la drogue» doit être perçue comme une laborieuse tentative d'appliquer la loi avec efficacité ou tout du moins de sembler le faire en usant de moyens de surveillance et de maintien de l'ordre purement techniques ou même militaires. C'est la justice pénale qui définit, sélectionne, documente et règle le crime. Cela sous-entend une certaine liberté dans l'interprétation des définitions juridiques de termes comme suspect, délit, etc. De plus en plus, le système de justice pénale précise donc lui-même à la fois la nature du problème de la criminalité et les types de solutions possibles (p. 128). 
Ainsi, au lieu d'en être la solution, le système de justice pénale fait partie du problème de la criminalité. Il échoue donc au niveau des buts qu'il s'est fixés. De plus, lorsque l'État inflige des souffrances, cela a des conséquences néfastes qui menacent de devenir incontrôlables. Plus important encore: le système est fondé sur un mode de compréhension fondamentalement erroné. Par conséquent, il est inutile d'essayer de le rendre plus efficace ou plus équitable.

\section{L'ABOLITIONNISME EN TANT QUE STRATÉgIE POLITIQUE}

A l'origine on a mis au point une stratégie politique inspirée de l'expérience des groupes de réforme carcéral dans leur lutte pour obtenir une réforme pénale et sociale. C'est dans ce contexte qu'est apparue la stratégie abolitionniste de la «réforme négative (Mathiesen, 1974, 1986). Cette stratégie consiste à refuser constamment d'offrir des solutions positives. Elle se limite à proposer des réformes ouvertes, «inachevées» et "négatives" comme l'abolition de certaines parties du système carcéral. Cela se traduit par une nécessité de les présenter à l'aide d'une terminologie étrangère au discours de la justice pénale.

Plus récemment, on s'est intéressé à des solutions positives à la peine. Diverses propositions ont été avancées par les abolitionnistes et d'autres en vue de décentraliser ou même de démanteler complètement le système pénal actuel et créer ainsi des formes de «justice informelle» pour compléter ou remplacer ce système.

Néanmoins, leur mise en œuvre soulève également beaucoup de questions quant à leurs effets contradictoires. On peut venir à grossir le filet tout en amenuisant ses mailles, ou encore à reculer les frontières entre l'intervention pénale officielle et d'autres manières informelles de contrôle social tout en les rendant plus incertaines, ce qui camoufle le caractère coercitif des autres modes d'intervention proposés (Abel, 1982 ; Cohen, 1985).

Pour une réforme en profondeur du système pénal, il ne suffit pas de trouver des alternatives originales. Une restructuration intégrale des rapports de pouvoir s'impose. Certains auteurs ont soutenu alors une stratégie de "réforme négative» visant à changer non seulement le système de justice pénale, mais aussi le système capitaliste répressif, étape par étape (Mathiesen, 1986). 
Une réforme en profondeur du système pénal présuppose une métamorphose complète de la structure de pouvoir en place ainsi que de la culture dominante. Il n'existe cependant aucune organisation sociale adéquate pour soutenir une réforme radicale de la politique du châtiment. En effet, il semble n'y avoir aucun support social immédiat pour l'édification spontanée d'une stratégie de contrôle social progressiste, et encore moins abolitionniste (Matthews, 1987, p. 389). Les abolitionnistes ont tendance alors à se référer à la réémergence des sous-cultures des nouveaux mouvements sociaux avec leur propre infrastructure d'interaction et de communication, ainsi qu'avec leur nouvelle éthique de solidarité, de responsabilité sociale et de compassion (Steinert, 1986, p. 28-29; Christie, 1982, p. 75-80). Comme le soutient Harris (1987, p. 11), la précarité d'à peu près toutes les propositions de réforme s'explique par l'incapacité à sortir des sentiers battus. Pour explorer une vision alternative de la justice, il faut des «philosophies, paradigms, or models that transcend not only conventional criminological and political lives, but also natural and cultural boundaries and other limiting habits of the mind» (op. cit., p. 11). Toujours selon Harris, il faut réussir et mettre à contribution une grande variété de points de vue sur la manière de créer un monde meilleur et un avenir plus souriant afin de pouvoir repenser notre approche à la criminalité et à la justice. Les nouveaux mouvements sociaux, notamment les mouvements de femmes, ont mis en relief les faiblesses inherentes ou les biais des postulats de la criminologie, des cadres conceptuels, de la méthodologie et de la moralité tacite (Gelsthorpe et Morris, 1990). Néanmoins, les rapports entre certains courants du féminisme et l'abolitionnisme posent encore quelques difficultés (voir Van Swaaningen, 1989).

\subsection{L'abolitionnisme et le contrôle de la criminalité}

L'abolitionnisme favorise une approche structurelle à la prévention de la «négativité sociale», à savoir le redressement des situations problématiques en prenant les problèmes sociaux, les conflits et les troubles au sérieux sans pour autant les traiter comme s'ils étaient des «crimes». L'abolitionnisme prône donc une politique sociale plutôt qu' une politique de contrôle de la criminalité. Les problèmes de drogue, par exemple, seraient traités comme des problèmes de santé mentale; la violence, en termes de pathologie sociale; et les atteintes à la propriété comme trahissant des questions d'ordre économique.

L'abolitionnisme appelle la «décriminalisation», la dépénalisation, la «déstigmatisation», la décentralisation et la «déprofessionnalisation" ainsi que la mise en place d'autres moyens informels, (semi-) 
autonomes et interactifs pour faire face aux problèmes sociaux. Il prend les conflits au sérieux sans les considérer comme des «crimes». On peut aussi bien qualifier les situations problématiques de troubles sociaux, problèmes ou conflits engendrés par une négligence ou par «accident» au lieu de faire appel aux qualifications de préméditation ou d'intention criminelles. Ce qu'il faut, c'est un large éventail de réponses possibles sans a priori sur les éventuelles intentions et responsabilités criminelles.

Comme nous l'avons vu, l'abolition de la peine de prison et, a fortiori, du système pénal, réclame un nouvelle manière de penser les différentes façons possibles de traiter des situations problématiques en les envisageant comme des problèmes sociaux, des conflits, des troubles, des accidents, etc. En outre, elle demande de revoir le concept de peine et de suspendre, au moins partiellement, la logique de la culpabilisation et de répression. Il faut aussi élaborer de nouvelles façons de gérer la "déviance». Si l'on accepte de ne pas voir entièrement les choses en termes de culpabilité individuelle, de responsabilité et de punition, les «crimes» deviendraient des «conflits", des «accidents» ou des «situations problématiques» devant être affrontés d'une manière plus raisonnable et humaine, a travers des formes de gestion des conflits qui ne soient pas exclusivement axées sur l'individu et limitées par le cadre du droit pénal, aussi bien dans les textes que dans la réalité (Steindert, 1986, p. 30). C'est pourquoi les abolitionnistes privilégient des solutions extra-pénales ou «autonomes» aux problèmes sociaux comportant des infractions à la loi. Dans la perspective abolitionniste, il s'agit désormais de trouver des types de sanctions plus «interactives", «populaires» ou «socialistes» pour remplacer le recours à la prison (Garland et Young, 1983).

Cette façon de voir la criminalité et son contrôle est bien entendu loin de faire l'unanimité. La perspective constructiviste des abolitionnistes est parfois tenue pour naïve et idéaliste. En pratique, cependant, l'approche abolitionniste s'avère réaliste, car, elle conçoit les problèmes et les conflits sociaux comme inhérents à la vie sociale et affirme qu'il est illusoire de penser que le système de justice pénale puisse nous protéger efficacement contre ce type d'événements maiheureux. Dès lors, il paraît plus raisonnable de traiter les problèmes de manière pragmatique au lieu de les aborder à travers le prisme de la culpabilité et du châtiment. Pour prévenir et contrôler efficacement des situations et des comportements inacceptables, il faut disposer d'une variété de réponses sociales. Le système de justice pénale n'en est qu'une, et ses interventions ont une valeur plus symbolique que 
pratique. Avec un peu d'imagination sur le plan social, technique et organisationnel, la «criminalité » pourrait être affrontée de manière bien plus humaine pour les personnes directement concernées. On pourrait mettre ne place et institutionnaliser diverses procédures où les problèmes sociaux ou conflits, les situations ou comportements problématiques pourraient être traitếs par le biais de la négociation, de la médiation et de l'arbitrage, à des niveaux intermédiaires.

Dans le cas des crimes les plus courants, qui en représentent du reste la vaste majorité, les poursuites criminelles sont tout simplement redondantes. Il est indubitable que les personnes directement visées en tirent peu ou pas profit.

On peut éviter de recourir à l'emprisonnement même dans les cas où un individu est devenu un poids inacceptable pour sa famille ou sa communauté. Il est possible de parvenir à des ententes ou à des sommations restreignant, sur une base temporaire ou permanente, l'accès à certains lieux, personnes ou situations. Reste le problème des individus véritablement mauvais ou fous. Dans ces cas, relativement peu nombreux, et, en dernier ressort, la privation de liberté peut s'avérer inévitable, tout au moins dans l'état actuel des choses. C'est une décision exceptionnelle qui devrait être prise uniquement comme mesure de neutralisation et appliquée de manière humaine, comme le serait n'importe quelle autre décision moralement discutable dans une situation problématique. Néanmoins, même dans ces cas, il serait préférable de rechercher des options plus équitables et plus humaines reposant sur l'entraide, le bon voisinage et un esprit communautaire, au lieu de continuer à s'en remettre aux solutions proposées par les bureaucrates, les professionnels et un Etat centralisateur.

L'accusation d'inhumanité et d'irrationalité que l'on porte à l'endroit de la solution carcérale est aussi valable aujourd'hui qu'il y a vingt ou soixante-dix ans. C'est ce qui conduit Cohen à suggérer trois stratégies corrélées, à savoir : tout d'abord, cultiver une attitude ouverte à l'expérimentation et encourageante par rapport aux options, aux innovations et aux expériences qui ont été tentées jusqu'à présent; ensuite, être sensible non seulement aux échecs, aux cooptations et aux stratagèmes malhonnêtes, mais aussi aux histoires de réussite (le critère de la réussite devrait et ne peut être qu'une approximation des valeurs préconisées) ; et, enfin, échapper aux griffes de la criminologie en élargissant les questions relatives au contrôle social au-delà du champ du système de justice pénale (par exemple à des systèmes de justice informels, des 
communes utopiques et des experimentations en auto-assistance) (Cohen, 1988, p. 131).

Dans les pays qui jouissent d'un système de sécurité sociale sophistiqué, comme les pays scandinaves ou les Pays-Bas, ces stratégies pourraient sembler plus réalisables, leur problème de criminali té étant moins grave et leur politique de contrôle de la criminalité traditionnellement plus modérée. Dans un climat pénal relativement clément où il existe déjà une politique pénale réductionniste et pragmatique, on peut concevoir plus facilement l'abolition du système pénal à plus long terme. Cependant, dans les pays où le nombre de détenus augmente astronomiquement et où les établissements pénitentiaires servent uniquement à «entreposer » certains individus pour les empêcher temporairement de récidiver, l'abolition de l'incarcération pose un problème indéniable. Au début des années soixante-dix, plusieurs commissions et groupes de travail sont parvenus à la conclusion que le système carcéral américain était tellement détérioré qu'aucune réforme ne pouvait plus le rescaper. Selon ces commissions, il convenait de trouver d'autres solutions aux problèmes de la criminalité Or, la population de détenus représentait alors un tiers des chiffres actuels. De nos jours, dans les conditions de surpopulation des prisons, ces critiques sont encore plus pertinentes. Les prisons sont des endroits qui font plus de mal qu'il n'est nécessaire ou légitime. En outre, ces etablissements favorisent l'augmentation de la violence sociale. Même aux États-Unis où les peines sont bien plus longues que, par exemple, aux Pays-Bas, $99 \%$ des condamnés finissent par se retrouver dans la rue. Il existe donc un besoin réel non seulement pour une réforme pénitentiaire, mais aussi pour une réforme pénale. La politique de contrôle de la criminalité actuelle revient à perpétuer les mêmes erreurs du passé. À long terme toutefois, il faudra bien changer d'orientation et briser ce cercle vicieux. Seules des solutions de rechange et plus humaines permettront de le faire.

L'abolitionnisme part du principe que les problèmes ou conflits sociaux sont inévitables du fait qu'ils sont inhérents à la vie sociale en tant que telle. Il faudra donc bien les régler d'une manière ou d'une autre. Au lieu d'en confier la solution à des experts cependant, il convient d'y faire face dans un esprit de réciprocité et de solidarité, conditions qui devront être créées par une action sociale et politique.

La question pressante reste, bien entendu, celle de savoir comment y parvenir. Pour commencer, il ne faut pas s'attendre à ce qu'une seule solution règle le problème. Pour tenir compte de la diversité des phénomènes sociaux pertinents, il faut envisager une grande variété de formes 
de régulation sociale qui ne sont pas situées dans l'État ou encadrées par celui-ci, mais qui fonctionnent de manière semi-autonome et constituent des types de règlement des différends et conflits alternatifs, progressifs et émancipateurs.

La profonde insatisfaction à l'égard du système pénal actuel et, plus généralement, du système juridique, engendre un interêt croissant pour des formes «autonomes» de résolution des conflits et des différends. D'autres «styles de contrôle social» (Black 1976, p. 4-5) sont vus alors comme prometteurs et attrayants dans la mesure où ils permettent aux parties concernées de participer davantage au règlement du différend ou du problème. L'objectif est la compensation plutôt que les représailles; la réconciliation plutôt que l'octroi d'un blâme. À cette fin, le système de justice pénale doit être décentralisé et des tribunaux de quartiers mis sur pied pour compléter ou suppléer les autres.

Pour l'abolitionnisme, la question du crime comme problème social et comme objet d'analyse scientifique doit être entièrement repensée. Avec son élargissement aux sphères académiques et sa promotion au rang de perspective théorique, l'abolitionnisme a étendu son champ d'intérêt du système carcéral au système pénal, s'engageant de ce fait dans des analyses critiques du discours pénal et, plus particulièrement. des concepts de crime et de peine, etc.

\section{L'ABOLITIONNISME COMME PERSPECTIVE THÉORIQUE}

En tant que perspective théorique, l'abolitionnisme propose un nouveau paradigme qui a une facette "négative» ou critique et une facette "positive» ou constructive. Dans sa dimension critique, l'abolitionnisme est profondément engagé dans une mise en cause du système de justice pénale et de la solution que ce système propose au problème de la criminalité, à savoir la prison. Dans sa dimension constructive, l'abolitionnisme, sur la base de cette critique, propose une nouvelle approche à la criminalité et à la peine à la fois sur les plans théorique et pratique. Ainsi, l'approche abolitionniste est fondamentalement réflexive et (dé) constructiviste. Nous examinerons tout d'abord l'aspect critique de l'abolitionnisme avant d'aborder brièvement son aspect constructif.

D'un point de vue abolitionniste, la prétention qu'a le système de justice pénale de protéger les gens en prévenant et en contrôlant la criminalité semble outrancière. De plus, l'idée de contrôler la criminalité 
par une intervention pénale pose un problème d'éthique, les gens étant utilisés comme moyens de «dissuasion", dans des démonstrations de pouvoir et de domination. Le châtiment est perçu comme une forme de violence qui se reproduit d'elle-même. La pratique pénale consistant à blâmer des individus pour leurs intentions supputées, c'est-à-dire les traiter de mauvaises personnes, puis les punir et les abaisser en consequence, est dangereuse car on reproduit de la sorte les conditions sociales propices à la récidive. La dégradation morale des personnes et leur ségrégation sont des pratiques d'autant plus pernicieuses que la logique de l'exclusion aboutit à une différentiation de sexe, de race, de classe, de culture ou de religion.

Pour les abolitionnistes, les politiques de criminalité actuelles sont irrationnelles. Car elles partent des postulats que la criminalité est un problème social propre à certains individus qui, pour quelque raison, se fourvoient; que la criminalité est un problème dont le contrôle revient à l'État et à son système de justice pénale, et que le droit pénal et le châtiment ou le traitement des infracteurs constituent des moyens appropriés pour contrôler la criminalité (Steinert, 1986). La politique de contrôle de la criminalité est fondée sur une démarche qui tend à prendre la pars pro toto. Cette politique est alors typiquement construite à partir d'incidents dramatiques. On considère aussi que l'important est de réussir sur le plan microscopique, sans se préoccuper du plan macroscopique. Selon Wilkins, cependant, nous devons tenir compte non seulement de l'acte criminel lui-même, mais aussi de l'environnement dans lequel il a eu lieu (Wilkins, 1984).

\subsection{Le point de vue abolitionniste sur la notion de crime}

Les abolitionnistes considèrent la criminalité comme étant essentiellement la résultante de l'ordre social et sont convaincus que le châtiment n'est pas la bonne réaction. Ils préconisent au contraire un minimum de coercition et d'intervention dans les vies personnelles des individus concernés, ainsi qu'un maximum de soins et de services à tous les membres de la société. L'approche actuelle du contrôle de la criminalité, la définition du crime et la justification du châtiment sont «systématiques", c'est-à-dire fondées sur une doctrine instrumentaliste et confinées aux limites du système de justice pénale.

D'un point de vue abolitionniste, ces questions doivent être fondamentalement redéfinies et resituées dans le contexte plus vaste de la vie quotidienne. C'est là qu'embraie l'aspect constructif de l'abolitionnisme. Les abolitionnistes prétendent que le concept de «crime» n'a 
aucune dimension ontologique; en d'autres termes, que ce phénomène appelé «crime» n'existe pas. En fait:

«The very form of criminal law, with its conception of «crime» (not just the contents of what is at a given time and place defined into that category, but the category itself) and the ideas on what is to be done about it, are historical «invention,» (Steinert, 1986), p. 26)

Le «crime» est une construction sociale et doit être analysé comme un mythe de la vie quotidienne (Hess, 1986). En tant que mythe, la notion de crime sert à maintenir les relations de pouvoir politique et à légitimer l'expansion de l'appareil de contrôle de la criminalité, de même que l'intensification de la surveillance. Elle justifie l'inégalité et la privation relative. L'opinion publique est détournée des problèmes plus graves et des injustices. Ainsi, plus les problèmes sociaux sont grands, plus on a besoin du mythe du crime (Hess, 1986, p. 24-25).

Cependant, il ne suffit pas de se débarrasser du concept de crime (Hulsman, 1986). Il faut aussi se défaire des théories sur la criminalité. Comme Quensel (1987) l'a souligné, les théories sur la criminalité assoient leur plausibilité sur une «structure profonde» préexistante et qu'elles renforcent de ce fait. Citons, à titre d'exemples, deux éléments de cette «structure profonde ": l'idée que la dangerosité et la méchanceté sont inhérentes au crime; et l'idée que le contrôle de la criminalité est une action inspirée par un système de valeurs, visant à prendre des mesures contre ce mal (p. 129).

Les abolitionnistes affirment que le problème essentiel n'est pas d'expliquer mais plutôt de comprendre le crime comme un événement social. Nous n'avons donc que faire d'une meilleure théorie de la criminalité. Ce qu'il nous faut, c'est un regard plus puissant sur ce qu'est le crime. Il ne s'agit pas de nier qu'il se passe toute sorte d'evénements regrettables, des troubles et des conflits plus ou moins graves qui peuvent causer de la souffrance, de la douleur ou des dégâts à un degré plus ou moins élevé. Il faut les prendre au sérieux, cela va sans dire, mais sans les qualifier de «crimes » et, surtout sans avoir recours au droit pénal. Lorsque l'on apprécie pleinement la complexité du «crime», comme un phénomène socialement construit, recourir à une solution aussi simpliste que le châtiment devient problématique (Steinert, 1986).

Spector (1981) argumente que, lorsqu'un individu offense, dérange ou blesse d'autres personnes, il existe diverses formes de désapprobation sociale pour remédier à la situation. Le problème peut être abordé comme une maladie, un péché ou même un crime. Toutefois, on peut 
aussi considérer le cas comme un conflit personnel entre l'agresseur et l'agressé ou définir la situation de manière administrative et réagir, par exemple, en refusant un brevet, un permis, ou en accordant un avantage social ou un dédommagement. Nos images, notre langage, nos catégories, nos connaissances, nos croyances et nos peurs par rapport aux fauteurs de troubles changent constamment. Néanmoins, la notion de crime continue à occuper une place centrale dans notre esprit pour désigner les actes de ceux qui nous dérangent (1981, p. 154). Spector suggère que peut-être «we pay too much attention to crime because the disciplines that study trouble and disapproval - sociology and criminology - were born precisely in the era when crim was at its zenith » (Ibidem; voir aussi Quensel, 1987).

Le concept de «crime» occupe une place importante dans le sens commun et a une influence irréfutable sur celui-ci. Lorsque l'on attire l'attention du public sur une catégorie donnée d'événements que l'on qualifie de «crimes», on réclame presque automatiquement une punition. Le «châtiment » est donc perçu comme la réaction évidente et appropriée au «crime».

\subsection{Le point de vue abolitionniste sur la peine}

Les abolitionnistes ne partagent pas la confiance que l'on place de nos jours dans la compétence du droit pénal à contrôler la criminalité. Ils réfutent catégoriquement l'utilité de la peine et affirment qu'il ne peut y avoir de justification valable pour y recourir, surtout qu'il existe d'autres options pour faire respecter la loi. Ils rejettent la justice criminelle comme une «idée absurde». Selon eux, il est ridicule d'affirmer qu'une souffrance peut ou doit même être compensée par une autre infligée par l'État. Selon eux, la solution de la prison porte atteinte à la qualité morale de la vie dans la société dans son ensemble. C'est pourquoi il faut se détourner de l'orientation donnée par la justice pénale et la remplacer par une orientation visant à réduire la souffrance et la douleur (Steinert, 1986, p. 25). Christie (1982) s'est plus particulièrement attaqué aux justifications traditionnelles de la peine. Il critique la théorie de la dissuasion pour ses définitions hâtives des concepts, parce qu'elle se prête mal au processus de réfutation empirique d'hypothèses et parce qu'elle donne à la pratique courante de la peine une fausse légitimité, à une époque où infliger de la souffrance est, à tout autre égard, problématique. Il critique aussi la théorie néo-classique du «juste dû » (just deserts), car elle justifie et objective la peine, blâme l'infracteur et ignore la victime. La conception de la justice y est réduite à sa plus simple expression, et on transmet un «message caché » qui nie 
toute légitimité à un ensemble d'alternatives qui devraient pourtant être prises en considération. Cependant, Christie ne se contente pas de critiquer les «prétendues justifications» de la peine. Il adopte à l'égard de cette dernière une position décidément morale qu'il appelle «rigorisme moral ». Il choisit à dessein les termes «moralisme» et « rigorisme » qui ont été associés principalement jusqu'à présent aux défenseurs «de la loi et de l'ordre" et des sanctions pénales plus sévères. Sa position «rigoriste» aboutit toutefois à un résultat différent et l'amène à affirmer qu'il n'y a aucune raison de croire que le degré de douleur que l'on inflige de nos jours soit juste ou naturel, et qu'il n'est aucune autre position défendable que celle de lutter pour la réduction des souffrances infligées par l'être humain sur terre. La peine étant synonyme de souffrance, limiter cette dernière revient à réduire automatiquement le premier.

Plus récemment, Christie et Mathiesen ont tous deux avancé que l'expansion du système carcéral soulève des questions d'éthique et de politique générale telles que: quels pourraient être les effets de toutes les peines prises dans leur ensemble? Quel serait le niveau admissible de châtiment dans la société ? Quel serait le niveau acceptable de la population carcérale dans un pays? Comment devrions-nous traiter nos semblables? Et, enfin, comment affronter le probleme de la criminalité ? (Christie, 1986; Mathiesen, 1986).

Cependant, dans le discours populaire aussi bien que juridique, le «crime» et la «peine» sont toujours perçus comme «independent species - without reference to their sameness or how continuity of both depends on the character of dominating institutions " (Kennedy, 1974, p. 107). Il ne faut pas oublier toutefois que le crime n'est qu'une sorte de violation des normes parmi d'autres et que la peine n'est qu'une façon parmi d'autres de sanctionner ces transgressions (p. 108). La criminologie doit aussi se débarrasser des théories de la peine qui accordent un statut d'évidence à celle-ci ou établissent un lien direct entre la violation des lois pénales et le châtiment. La notion de "peine " (châtiment) étant traditionnellement associée à la lutte du «bien contre le mal ", tout effort visant à modifier les perceptions populaires du "crime» et du «contrôle de la criminalité » doit passer par une révision de ces deux concepts: ceux de «crime» et de «peine». 


\section{LA NOTION DE REDRESSEMENT}

Nous devons examiner maintenant les liens qui existent entre le crime et le châtiment ainsi que leurs effets combinés. Le crime et le châtiment sont étroitement liés à la «négativité sociale" (Baratta, 1986), aux développements destructeurs dans la société moderne, surtout parce qu'ils atteignent ses membres déjà les plus vulnérables. Pour formuler une politique de réforme pénale crédible, le crime et le châtiment ne devraient pas être considérés comme action et réaction mais comme une spirale ascendante de torts (Pepinsky, 1986).

J'ai parlé ailleurs du concept de «redressement» (redress) comme alternative aux concepts de «peine» (châtiment) et de «crime» (de Haan, $1990 ; 1991$ ). Ce concept, qui peut paraître désuet, véhicule tout un assortiment de significations. Le Concise Oxford Dictionary ( $6^{\mathrm{e}}$ édition, 1976, p.937) en donne plusieurs dont en voici quelques-unes: placer en ligne droite ou remettre la situation en bon état; remédier ou supprimer un mal quelconque; redonner une forme droite; réparer; rectifier les erreurs, les torts ou les maux causés; corriger; amender; réformer le mauvais état des choses; réparer une action; racheter une faute: sauver; délivrer de la misère; ramener une personne à l'état normal, au bonheur, à la prospérité ou au droit chemin; compenser correctement une personne en lui obtenant ou (plus rarement) en lui donnant une réparation pour un tort ou une perte subie; enseigner, instruire ou redresser l'erreur par la raison. La diversité de ces significations explique parfaitement pourquoi le concept de «redressement» répond mieux que tout autre aux besoins impérieux de la criminologie critique et constitue une alternative aux concepts de «châtiment » et de «crime». Sommairement, ses avantages sont les suivants:

a) La notion de «redressement » englobe presque toutes les réactions imaginables à un événement individuel, collectif, structurel, matériel ou immatériel.

b) Elle implique que la réaction est obligatoire, sans prédéfinir l'événement comme un crime, une maladie ou quoi que ce soit d'autre.

c) Elle invite à analyser l'évênement avant de décider ou de choisir la réaction qui convient.

d) En tant que concept avec des origines anciennes, il encourage à étudier les formes historiques et anthropologiques de règlement de contentieux et de conflits pour trouver une solution rationnelle au problème. 
Parler de redressement, c'est noter simplement qu'un événement indésirable a eu lieu et qu'il faut faire quelque chose en ce qui le concerne. Cela n'oriente aucunement le type de réaction qu'il faut adopter. De plus, on n'a pas besoin de définir d'une manière particulière les événements indésirables. On invite au contraire à une discussion ouverte sur la nature regrettable de l'événement et sur la réaction qu'il convient de choisir. C'est donc la réaction rationnelle par excellence. On réclame ainsi la mise en place d'une procédure quelconque plutôt qu'une démarche. La notion de châtiment qui est inévitablement associée à celle de «crime " devient de ce fait l'objet d'un débat rationnel. Aussi, prôner le «redressement», c'est inviter à un «véritable dialogue» (Christie, 1982, p. 11) ou encore à un «discours pratique» (Habermas, 1983).

\subsection{La notion de redressement et le discours pratique}

Le «discours pratique» (practical discourse) s’intéresse à la validité ou au bien-fondé de l'action et des normes et valeurs qui permettent de porter des jugements. Le point de départ du discours pratique est une perturbation active des attentes normatives. Lorsqu'une personne est amenée à mettre en cause la validité des normes ou des valeurs qui font partie d'un jugement ou d'un événement, survient un conflit moral qui devient matière à un discours pratique. Celui-ci consiste à trouver les moyens de résoudre un conflit moral pertinent de manière rationnelle, c'est-à-dire sans recourir à la force, excepté celle du meilleur argument.

Les participants doivent tous avoir les mêmes possibilités de prendre part à la discussion; et cette égalité doit être à la fois formelle et effective. Non seulement doivent-ils pouvoir intervenir dans le débat, mais encore doivent-ils pouvoir exprimer leur indignation, exposer leur point de vue, donner leur version, formuler leurs critiques et proposer leurs avis sur les solutions possibles. Le discours pratique n'est pas purement formel en ce sens qu'il ne s'éloigne pas du contexte. En réalité, il dépend de son thème précis pour sa propre justification. Le discours pratique nous renvoie alors au problème concret, mais il ne se réduit non plus à ce problème. S'il n'y avait pas de conflits moraux au sein d'un groupe social donné liés à une situation particulière, le discours pratique n'aurait tout simplement pas lieu d'être. Le discours pratique dépasse alors les limites de la situation concrète, du groupe ou de la communauté concernés, car son but est de parvenir à des conclusions généralisables et fondées sur des principes. On tient compte des éléments concrets disponibles ou soumis par les participants. C'est donc 
un discours plutôt exhaustif que restrictif. On estime en effet qu'une entente qui ferait abstraction des individus concernés ou des détails concrets de leur existence ne peut guère, dans la perspective du discours pratique, être considéré comme une forme d'argumentation rationnelle. Ce type de résultat ne rendrait justice ni aux personnes intéressées ni au cas concret. Lorsque l'on débat des normes et des valeurs qui devraient nous guider et des règles qui devraient organiser notre existence, il faut parler des intérêts particuliers, des antécédents, des loyautés, des capacités, des peurs et des aspirations des membres du groupe en question (Fay, 1987, p. 183). Les antécédents et les personnalités des personnes visées ainsi que le contexte de l'interaction sont des éléments essentiels dans le discours pratique. C'est seulement si l'on en tient pleinement compte que le résultat reflétera les idiosyncrasies des participants au débat et sera donc acceptable pour eux. En d'autres termes, la «bonne » réponse à un problème, dans un discours pratique, ne peut être dissociée du contexte spécifique propre aux individus pour qui ledit problème apparaît en premier lieu. En tenant compte du contexte, on obtient une quantité d'explications jusqu'à ce que les arguments et, éventuellement, le résultat commencent à revêtir un sens.

Dans le discours pratique, on est bien entendu obligé de faire face aux notions de «devoir des comptes» (accountability), de « responsabilité », de "culpabilité » et autres concepts juridiques, tous très problématiques. Néanmoins, en favorisant une interprétation plus complexe du comportement, des situations et des événements sociaux, on pourrait éviter l'image simpliste que se font actuellement le droit pénal et la justice criminelle de l'être humain et de ses activités. En remettant les choses dans leur contexte, on pourrait remplacer le caractère dichotomique de la justice criminelle (Christie, 1986, p. 96) par un continuum. Les participants seraient poussés à appréhender la complexité qui en-

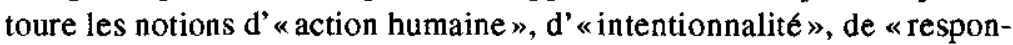
sabilité" et de "culpabilité» et obligés à y faire face plutôt que de les réduire à des proportions commodes en appliquant la logique binaire du droit pénal. En abandonnant les dichotomies simplistes du droit pénal et en introduisant des significations différentielles, justice sera peut-être enfin rendue à la complexité des agissements humains et des événements sociaux. Un tel discours mettrait en avant un concept de « responsabilité sociale » ouvrant la voie à des interprétations qui feraient porter la responsabilité plutôt aux systèmes sociaux qu'aux individus (Christie, 1986, p. 97). Le discours pratique ne peut se tenir que lorsque certaines conditions sont réunies tant sur le plan individuel que social. 


\subsection{Les conditions humaines et sociales pour la réalisation du discours pratique}

Premièrement, il faut un minimum de compétence et d'assurance de la part des participants. "Les règles du discours éthique» (rules of discourse ethics) permettent à tous de participer à égalité au discours pratique. Toutefois, prendre la parole et exprimer son sentiment demandent un certain degré d'assurance qui n'est possible que si les personnes reçoivent une certaine reconnaissance sociale. La plupart des gens ont besoin d'être rassurés sur le fait qu'ils sont respectés comme membres compétents de la communauté et que leur contribution au débat sera prise au sérieux. Il faudra donc mettre en place des processus de socialisation de manière à donner à tous les mêmes chances d'acquérir la disposition, la compétence et le talent nécessaires pour participer à une argumentation morale pratique.

Deuxièmement, le discours pratique pouvant, de par sa nature, durer indéfiniment, tôt ou tard, il faudra tirer des conclusions et prendre des décisions. Dans un discours pratique amorcé en fonction de la question du redressement, le point de départ n'est pas la définition juridique de ce qui s'est passé puisque les règles de fond du droit refleteraient un consensus moral sur ce qui est bien ou mal. On part plutôt du principe ambigu qu'il est impossible et même guère souhaitable de parvenir à une entente parfaite sur des sujets moraux et juridiques. Cela permet une discussion ouverte dans laquelle il est présumé que l'éthique du discours garantit une assez grande complexité et une participation suffisante pour que le résultat soit valable, tant en ce qui concerne la substance que la procédure. On peut tout de même s'interroger sur la vraisemblance de trouver une solution lorsque la procédure est si ouverte. Le discours pratique entraînant, en principe, une discussion ouverte, il est évident qu'il faut prévoir une procédure de prise de décision pour arriver à une évaluation, des décisions, et, enfin, leur mise en œuvre.

Le discours pratique ne doit pas être considéré comme une option déjà bien déterminée qu'il suffit de choisir mais plutôt comme une orientation vers des processus d'apprentissage collectif. Après avoir réuni les conditions humaines et sociales nécessaires au discours pratique, il faudra procéder par étapes. L'éthique du discours donne des directives pour traiter rationnellement les dissensions d'ordre moral et résoudre les conflits normatifs de manière discursive au lieu de le faire par la force. Cela implique une prédilection morale pour certaines formes d'organisation sociale qui favorisent la participation équitable 
de tous et garantissent les mêmes possibilités d'acquérir la compétence et l'assurance nécessaires pour le faire. Toutefois, à elle seule, l'éthique du discours ne peut créer les conditions requises pour sa mise en vigueur. Dans la réalité, ces conditions sont notoirement absentes. Par conséquent, il est impossible de discuter des conflits moralement pertinents dans un discours pratique lorsqu'on cherche seulement la commodité ou lorsqu'il y a des déséquilibres structurels du pouvoir. Dans ce cas, il devient inutile de parler de principes moraux universels, et la solution des conflits moraux est décidée par la force, la violence ou l'humiliation et l'outrage. Dans ces circonstances, il y a peu de place pour la transformation sociale dans le cadre du discours rationnel. Les questions d'éthique sont réduites à des questions politiques dont les solutions sont décidées par la force. Une éthique de discours enracinée dans la conscience de la vulnérabilité humaine serait impensable. Une si morne réalité est, à l'évidence, bien éloignée des conditions humaines et sociales sans lesquelles il ne peut y avoir de discours pratique.

Dans les conditions plus civilisées de la démocratie sociale, les arrangements institutionnels sont tels que les conditions pour le discours pratique sont déjà plus ou moins en train d'être en place. La dimension critique de l'ethique du discours canalise notre conscience et notre réflexion sur les conditions matérielles et symboliques qui peuvent inhiber notre liberté de prendre part au discours et, plus particulièrement, d'exprimer nos préoccupations morales. L'apprentissage pratique et le changement social sont de la sorte encouragés. Pour continuer l'apprentissage pratique, il faut toutefois réunir au moins quatre conditions. Premièrement, il devrait être possible de prendre conscience de l'insuffisance des conditions pour la communication et pour la participation symétriques avec certains établissements, ainsi qu'avec les cadres conceptuels qui les légitiment. Deuxièmement, il devrait être possible d'articuler les expériences sous forme d'une critique des établissements existants et de leurs justifications. Troisièmement, il devrait être possible de porter ces expériences et leurs conceptualisations à l'attention du public éventuellement concerné. Quatrièmement, il devrait être possible aux participants d'expérimenter et d'employer des concepts alternatifs afin de mettre en question la pertinence des concepts normatifs (comme ceux de «responsabilite», de «culpabilité», etc.) jusque-là généralement acceptés sans hésitation.

$C^{\prime}$ 'est uniquement lorsque ces conditions sont réunies que les processus d'apprentissage pratique peuvent se poursuivre (Kunneman, 1982, p. 346-347). Il convient aussi de les réunir pour créer la mentalité particulière nécessaire à la réalisation de l'éthique du discours. Ce qu'il 
faut, c'est une disposition de base sur comment on doit traiter des conflits normatifs de la meilleure façon possible. 11 faut nous fier à la force des arguments tout en nous abstenant de recourir à la force ou à la violence.

Cela souleve la question de savoir comment interpeller le sens commun du public de façon à provoquer un changement des mentalités. Il existe de graves problèmes liés à l'élaboration d'une critique des théories actuelles de la criminalité (Quensel, 1987) et d'une critique des justifications des châtiments légaux (Baratta et Silbernagel, 1987). L'une des fonctions ou effets des «théories technologiques» actuelles de la justice pénale est d'éliminer les conceptions de la réalité qui diffèrent de Ia "normalité" entretenue et stabilisée par le système de justice pénale. On considère souvent que le respect de la loi et le contrôle de la criminalité sont des objectifs qui peuvent être atteints simplement en assurant un fonctionnement plus efficace et plus rentable du système de justice pénale. Or, ces théories de la justice criminelle ne sauraient être critiquées sur la base exclusive de la preuve empirique, car même lorsque cette «rationalité technologique» est appelée «rationalité partielle» ou même «irrationalité», elle reste «rationnelle», bien que d'une manière specifique et partisane (Baratta et Silbernagel, 1987, p. 38).

Aujourd' hui, dans le récent glissement vers la droite, on constate un grand besoin de restaurer la relation entre la moralité et le droit pénal, ce qui rend la tâche de faire passer un message alternatif encore plus difficile. Même certaines parties des mouvements sociaux progressistes, comme certains groupes du mouvement des femmes, comptent encore sur l'appui de l'appareil de justice criminelle pour étayer leurs convictions morales de façon symbolique.Pour cette raison, le soutien de l'opinion publique pour les arguments critiques en faveur d'une politique alternative de la criminalité, de la peine et de la réforme pénale ne sera pas acquis d'avance simplement parce qu'ils se justifient sur le plan rationnel. Sur une note plus optimiste, cependant, l'histoire de la réforme penale montre que des changements assez spectaculaires peuvent avoir lieu, malgré tout, dans la façon de percevoir la criminalité, les criminels et la peine. On peut donc en conclure que les changements sont au moins possibles, même s'ils dépendent, du moins en partie, de conditions sociales, économiques et politiques changeantes. L'évolution de nos idées sur la criminalité et la peine suit sa propre logique, dans une certaine mesure. Nous ne pouvons pas partir de rien mais devons faire laborieusement notre chemin à travers notre héritage historique. Le but d'une politique de redressement serait de «arrange it 
so that the conflict settling mechanisms themselves, through their organization, reflect the type of society we should like to see reflected and help this type of society come into being (Christie, 1982, p. 113). Les problemes ou conflits sociaux pourraient être absorbés pour constituer de précieux outils d'intégration sociale du monde réel et de prévention de la souffrance sociale.

\section{CONCLUSION}

L'innovation conceptuelle proposée ici ouvre les horizons à une politique du redressement visant l'élaboration et la mise en cuvre de procédures allant dans le sens de l'éthique mise en place par le discours pratique. Comme nous l'avons vu, les procédures criminelles actuelles sont inadéquates pour résoudre les conflits normatifs dans le cadre d'un discours rationnel. Afin de multiplier les chances de participer des personnes concernées, les procédures fondées sur les règles et conditions nécessaires au discours rationnel devraient donc être fixées en dehors du champ du droit pénal, soit dans le droit civil ou même dans le monde réel. Au lieu de la panacée dont le système de justice pénale se réclame le dépositaire et qui prétend résoudre les problèmes de contrôle de la criminalité, l'abolitionnisme cherche des solutions aux problèmes, aux conflits et aux troubles sociaux dans le contexte de la vie quotidienne, en prenant au sérieux les expériences de ceux qui sont directement concernés et en tenant compte de la diversité inhérente à la mosaïque sociale.

11 peut être utile de mettre l'accent sur les processus de participation à la définition des événements ou sur les processus de contextualisation des conflits, mais cela peut aussi donner des résultats problématiques. Au nombre des différentes réactions que la notion de «redressement» implique, certaines sanctions peuvent encore être nécessaires et doivent être soumises à des principes et à des contraintes juridiques. Pour ces raisons et par souci de «justice» (fairness), la forme juridique est toujours nécessaire pour l'instant. De la même manière qu'il faut une imagination sociologique pour garantir une discussion ouverte, il faut une imagination juridique pour pouvoir mettre un terme aux contentieux qui, sans cela, se poursuivraient indéfiniment, et laisser la place à une possibilité d'appel. 


\section{BIBLIOGRAPHIE}

ABEL, R. (éd.) (1982), The Politics of Informal Justice, vol. 1 et 2, New York, Academic Press.

BARATT A, A. (1986), "Soziale Probleme und Konstruktion der Kriminalität» Kriminologische Journal, Beiheft 1, p. 200-218.

BARATTA, A. et SILBERNAGEL, M. (1987), "Neue Legitimationsstrategien des Strafrechts und ihre Kritik als Realitätskritik ", Kriminologische Journal, p. 3249.

BIANCHI, H. et VAN SWAANINGEN, R. (éds.) (1986), Abolitionism: towards a non-repressive approach to crime, Amsterdam, Free University Press.

BLACK, D. (1976), The Behavior of Law, New York, Academic Press.

CHRISTIE, N. (1982), Limits to Pain, Oxford, Martin Robertson, 1982.

CHRISTIE, N. (1986) «Images of man in modern penal law », Contemporary Crises, vol. 10, p. 95-106.

COHEN, S. (1985), Visions of Social Control. Crime, Punishment and Classification, Cambridge, Polity Press.

COHEN, S. (1988), Against Criminology, New York, Transaction Books.

VAN DIJK, J., MAYHEW, P., KILLIAS, M. (1990), Experiences of Crime across the World. Key findings from the 1989 International Crime Survey, Boston, Kluwer.

FAY, B. (1987), Critical Social Science. Liberation and its Limits, Cambridge, Polity Press.

GARLAND, D. et YOUNG, P., (1983), * Towards a Social Analysis of Penality in D. Garland et P. Young (éds.), The Power to Punish. Contemporary Penality and Social Analysis, London, Heinemann, p. 1-36.

GELSTHORPE, L. et MORRIS, A. (eds.) (1990), Feminist Perspective in Criminology, Milton Keynes, Open University Press.

HAAN, W. de (1990), The Politics of Redress. Crime. Punishment and Penal Abolition, London, Unwin Hyman.

HAAN, W. de (1991), «Abolitionism and Crime Control: A Contradiction in Terms", in $\mathrm{K}$. Stenson et D. Cowell (sous la direction de), The Politics of Crime Control, London, Sage Publications, (p. 203-217).

HABERMAS, J. (1983), «Diskursethik - Notizen zu einem Begründungsprogramm *, in Moralbewubstein und Kommunikatives Handeln, Frankfurt A.M., Suhrkamp, p. 53-126.

HARRIS, K. (1987), « Moving into the New Millennium: Toward a Feminist - Vision of Justice w, The Prison Journal, vol. 67, p. 27-38. 
HESS, H. (1986), « Kriminalität als Alltagsmythos, Ein Plädoyer dafür, Kriminologie als Ideologiekritik zu betreiben", Kriminologisches Journal, vol. 18, Beiheft 1, p. 22-44.

HULSMAN, L. (1986), "Critical Criminology and The Concept of Crime", Contemporary Crises, vol. 10, p. 63-80.

KENNEDY, M. (1974), «Beyond incrimination ", in Reasons, C. (ed.), The Criminologist and the Criminal, p. 106-135.

KUNNEMAN, H. (1982), «Athenticiteit en communicatieve symmetrie», Kennis en Methode, vol. 6, p. 332-350.

MATHIESEN, T. (1974), «The Politics of Abolition. Essays», in Political Action Theory, London, Martin Roberson.

MATHIESEN, T. (1986), «The Politics of Abolition», Contemporary Crises, vol. 10, p. 81-94.

MATTHEWS, R. (1987), «Taking Realist Criminology Seriously», Contemporary Crises, vol. 11, p. 371-401.

MORRIS, M. (éd.) (1976), Instead of Prisons: A Handbook for Abolitionists, Syracuse, New York, Prison Research Action Project.

PEPINSKY, H. (1986), «A Sociology of Justice», Annual Review of Sociology, vol. 12, p. 93-108.

QUENSEL, S. (1986), «Let's Abolish Theories of Crime», in J. Blad, H. van Mastrigt et N. Uitdriks; (Eds.) (1987), The Criminal Justice System as a Social Problem: An Abolitionist Perspective, Rotterdam, Mededelingen van het Juridisch, Institut van de Erasmus, Universiteit, p. 123-132.

ROSHIER, B. (1989), Controlling Crime. The Classical Perspective in Criminology, Milton Keynes, Open University Press.

SCHEERER, S. (1986a), "Towards Abolitionism», Contemporary Crises, vol. 10, p. 5-20.

SPECTOR, M. (1981), «Beyond Crime: Seven Methods to Control Troublesome Rascals », in H. Ross, (éd.) Law and Deviance, Beverley Hills, Sage, p. 127-157.

STEINERT, H. (1986), «Beyond Crime and Punishment», Contemporary Crises, vol. 10, p. 21-39.

SWAANINGEN, R. VAN (1989), «Feminism and Abolitionism as Critiques of Criminology ", International Journal of the Sociology of Law, vol. 17, p. 287-306.

WILKINS, L. (1984), Consumerist Criminology, London, Heinemann.

ZBIGNIEV, L., PLATEK, M. et RZEPLINSKA, I. (1991) (sous la direction de), Abolitionism in History. On another way of thinking, Institute of Social Prevention and Resocialization, Warsaw University. 